\title{
The research on open loop actuator response of high speed vehicle based on constant speed and constant elasticity
}

\author{
Yong Liu ${ }^{1}$ a Yongbing Chen ${ }^{1}$ Wenkui $\mathrm{Li}^{1}$ and Jianhong Shi ${ }^{2}$ \\ ${ }^{1}$ Department of navigation, Naval University of Engineering \\ Wuhan, China \\ ${ }^{2}$ Department of control engineering, Naval Aeronautical and Astronautical University Yantai, China \\ a1974748219@qq.com
}

Keywords: high speed vehicle; open loop; actuator response; virtual flying; stability

\begin{abstract}
Based on a kind of pitch channel model of supersonic aircraft which is released by America air force research center, a kind of open loop testing method is proposed to testify the actuator effect of a kind of high speed aircraft. The difference between this type of aircraft and other traditional aircraft is that open loop response is unstable so it is not easy to do this kind of actuator test. So a kind of virtual flying condition is assumed to make the simulation can last for a longer time, also it can make the analysis of actuator effect to be more clear and simple. Also the physical meanings of this kind of unstable open loop response is analyzed.
\end{abstract}

\section{Introduction}

The open loop characteristics research of vehicle is the most valuable preliminary test after aircraft digital simulation model is established and a preliminary validation is reasonable $\mathrm{e}^{[1-5]}$. The main aim is to test dynamic characteristic of the rudder system, research the efficiency of the rudder angle, namely the influence of rudder deflection to attack angle. But the difference of open-loop characteristics of the hypersonic flight vehicle and no-hypersonic flight vehicle is that the open loop characteristic is unstable. So the digital simulation time of open loop characteristic is selected as $0.5 \mathrm{~s}$, not too long, rather than the simulation time of a hypersonic flight vehicle can select in $3 \mathrm{~s}$ or even longer. At the same time, in view of the tradition hypersonic flight vehicle, through the research of open loop characteristics, we can even draw a rudder slant to the stability of the attack angle ratio, namely the rudder. For hypersonic aircraft, because it is not stable, so the ratio doesn't exist, but it can be observed the divergence speed by digital simulation, so simulation results are still valuable. Starting point is based on the above in this paper. The divergence speed of attack angle is researched in canard steady cases based on hypersonic longitudinal motion model published by the U.S. air force research laboratory by selecting typical cases of the constant speed and constant elasticity, thereby standard the instability degree of from the canard to angle $\operatorname{attack}^{[6-10]}$.

\section{Model Description}

Considering the elastic shape structure, a kind of pitch channel hypersonic aircraft model built according to Lagrange equation is released by USA air force as followed:

$$
\begin{gathered}
\dot{V}=\frac{T \cos \alpha-D}{m}-g \sin \gamma \\
\dot{\phi}=-2 \varsigma \omega_{n} \phi-\omega_{n}^{2} \phi+\omega_{n}^{2} \phi_{c} \\
\dot{\gamma}=\frac{L+T \sin \alpha}{m V}-\frac{g \cos \gamma}{V} \\
\dot{\alpha}=q-\dot{\gamma}
\end{gathered}
$$




$$
\begin{gathered}
\dot{q}=\frac{M}{I} \\
\dot{h}=V \sin \gamma \\
\ddot{\eta}_{i}=-2 \varepsilon_{m} \omega_{m i} \dot{\eta}_{i}-\omega_{m i}^{2} \eta_{i}+N_{i}
\end{gathered}
$$

Where

$$
T=\bar{q} S\left(C_{T \phi} \phi+C_{T}+C_{T}^{\eta} \eta\right), D=\bar{q} S C_{D}
$$

And $V$ is speed, ${ }^{\gamma}$ is the speed angle, $\alpha$ is attack angle, $Q_{\text {is }}$ the attitude angle speed, $h_{\text {is }}$ the height. $\phi$ is the oil supplying factor, $\delta_{c}$ is the duck wing and $\delta_{e}$ is the lift rudder.

\section{Simulation settings of free flying}

In order to testify the rightness of model of hypersonic aircraft, choose a initial height as $h_{0}=85000 * 0.3048$, initial speed as $V_{0}=7846 * 0.3048$, initial attack angle as $\alpha_{0}=0.0174$, and other initial state as: $\gamma_{0}=0, q_{0}=0, \quad \eta_{10}=0.4588 * 0.3048 * 14.59, \quad \eta_{20}=-0.08726 * 0.3048 * 14.59$, $\eta_{30}=-0.03671 * 0.3048 * 14.59$

Setting the throttle opening of engine is the switching control law for the PID control law and diffusion control law, and selection

$$
\phi_{c}=k_{e t} e_{v}+k_{s e t} e_{v}+k_{d e} \dot{e}_{v}+k_{e t b} \frac{e_{v}}{\left|e_{v}\right|+\varepsilon_{e v}}, e_{v}>0, \quad \phi_{c}=-300, e_{v}<0
$$

Among them, $e_{v}=V^{d}-V, k_{e t}, k_{\text {set }} 、 k_{d e} 、 k_{e t b}$ and $\varepsilon_{e v}$ are for the adjustable positive number.The expectations speed is selected as $V^{d}=2391$, control volume is selected as $\delta_{c}=1 / 57.3, \delta_{e}=0$, the canard open loop dynamic characteristics of testing superb craft, setting $\dot{\eta}_{i}=0$,setting the flight time as 0.5 seconds.

\section{Numerical Simulation and result analysis}

With above model and air coefficients and set initial condition as above paragraph, write a program with $\mathrm{m}$ language in Matlab software, then the simulation can be done and simulation results can be shown as following figures. Selecting $\Gamma=0$ as flight test at a constant speed, the simulation results are as follows:

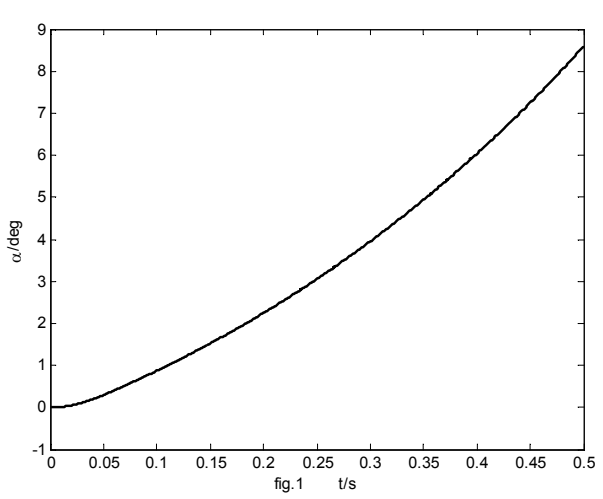

Fig 1 The curve of attack angle

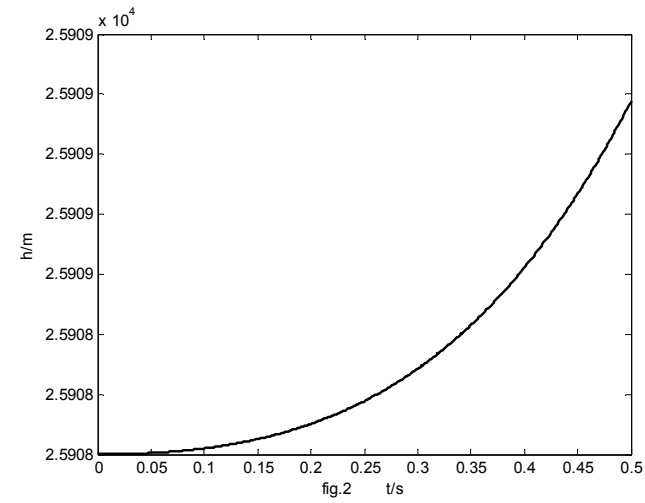

Fig 2 The curve of the height 


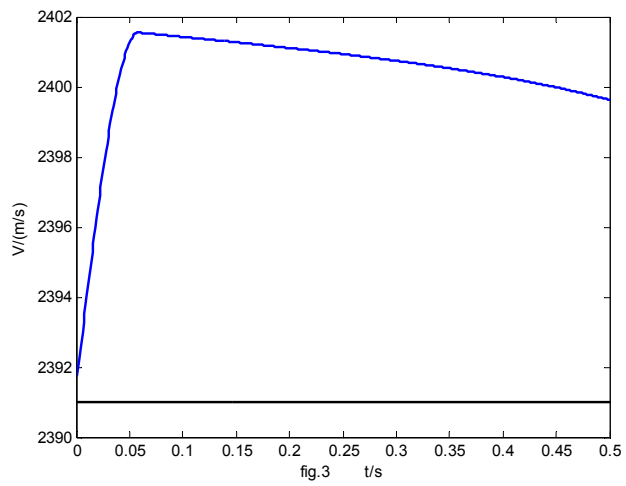

Fig 3 The curve of speed

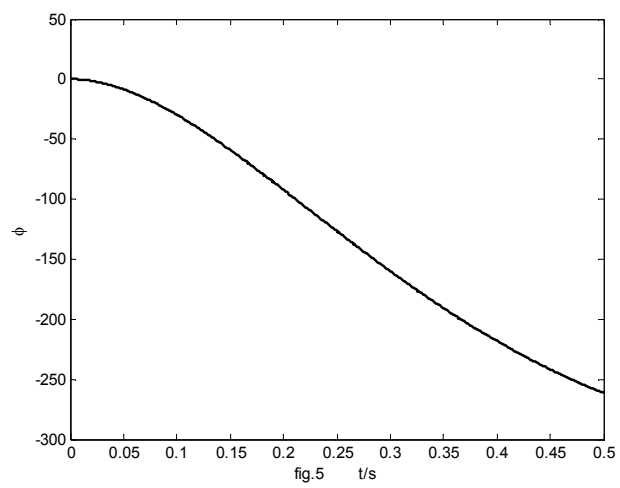

Fig 5 The curve of oil supplying factor

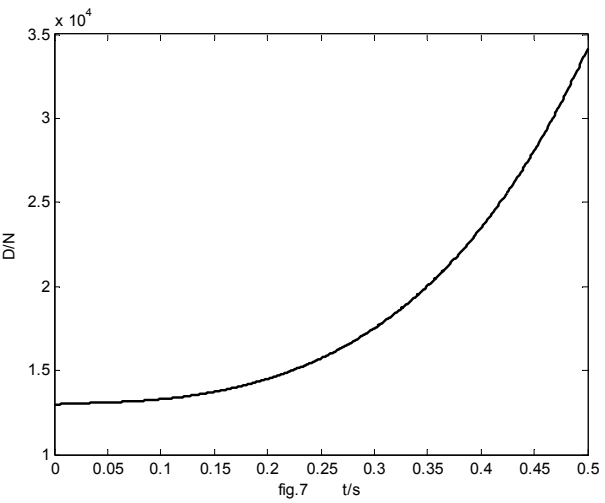

Fig 7 The curve of resistance

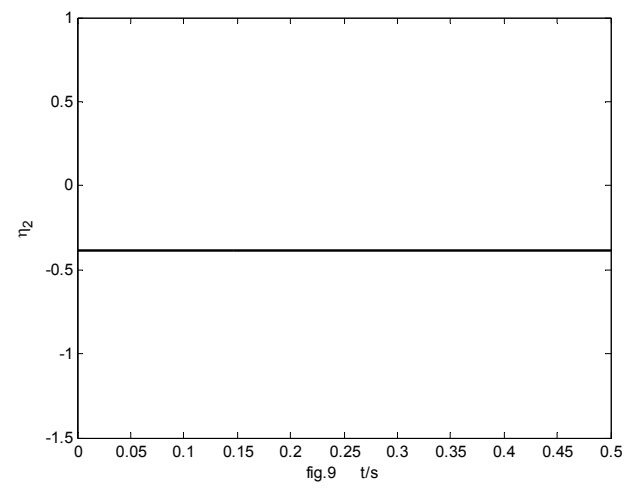

Fig 9 The state of second elastic shape

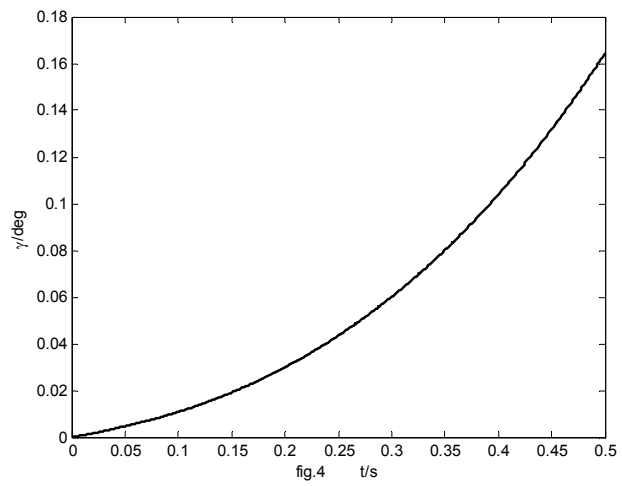

Fig 4 The curve of speed angle

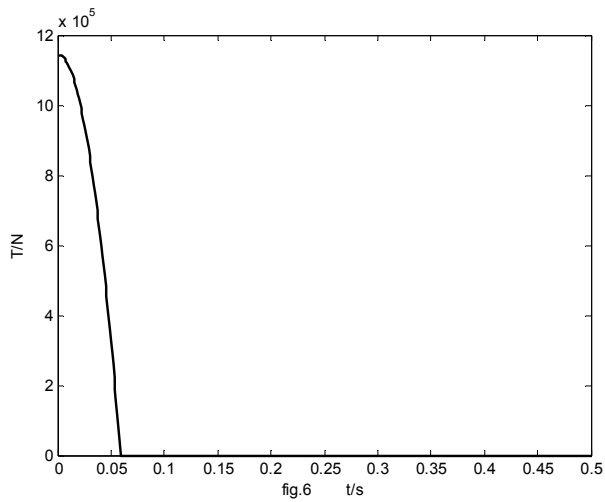

Fig 6 The curve of thrust

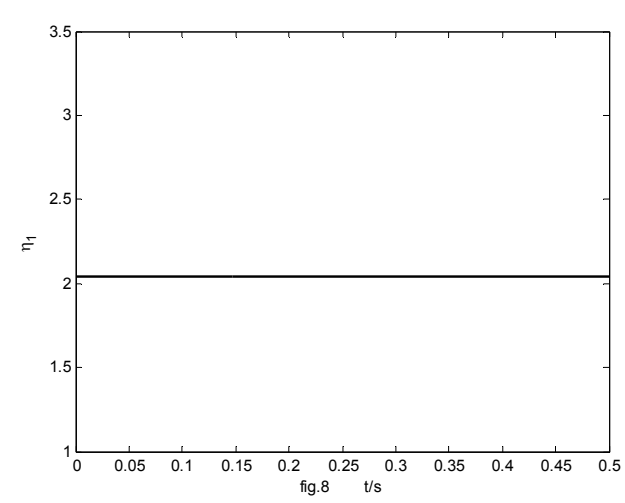

Fig 8 The state of first elastic shape

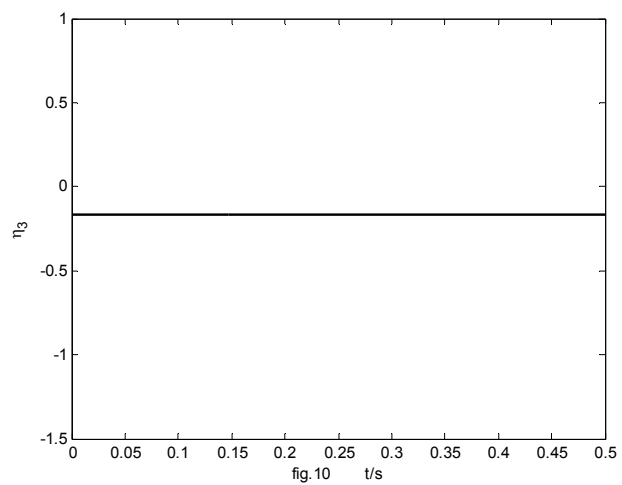

Fig 10 The state of third elastic shape 


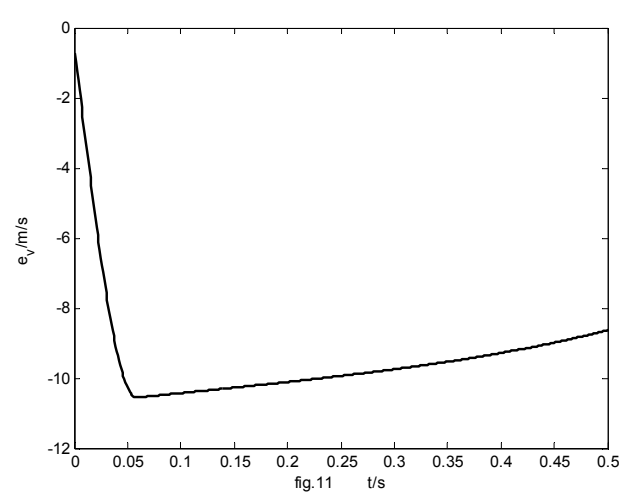

Fig 11 The state of speed error

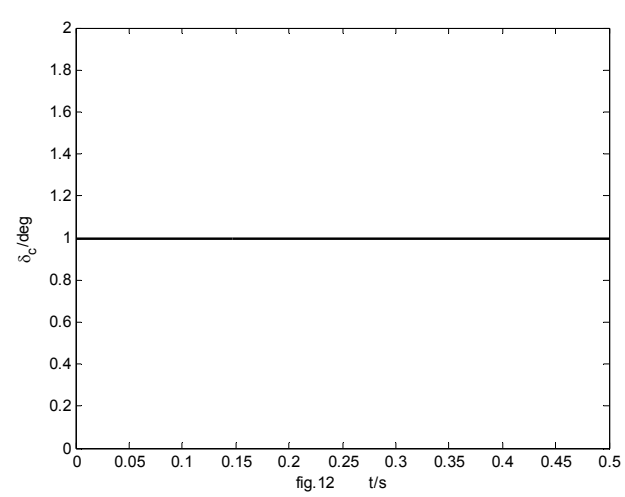

Fig 12 The curve of duck actuator

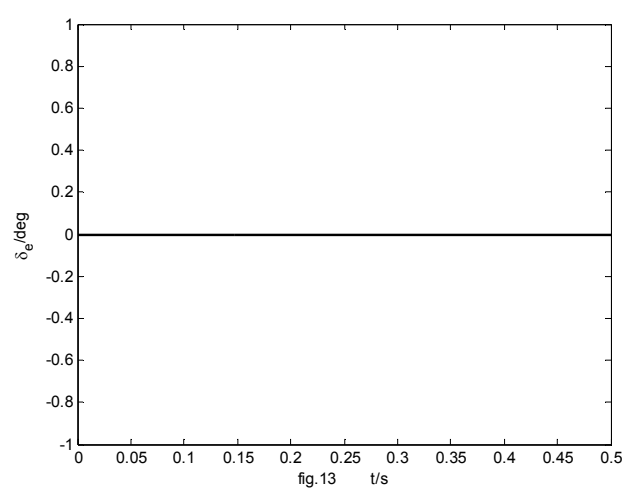

Fig 13 The curve of lift actuator

It can be seen from simulation that the elevation angle basic remains stable in the constant speed constant elasticity 0.5 seconds canard constant open loop flight. but the divergence of attack angle is too fast, the divergence 8 degrees of attack angle can be preliminary judged according to the graph.This is the place that is different from conventional aircraft at low speed, because open-loop characteristics of the low speed vehicle attack angle can remain stable for a short time. The attack angle of the hypersonic aircraft is static instability characteristics, it will bring greater difficulty and challenge for the design of its controller.

\section{Conclusion}

A kind of actuator open loop response is done with the help of numerical simulation technology although the pitch channel model is unstable without close loop control. The unstable features from the canard to attack angle is shown by setting open loop flight simulation of the hypersonic aircraft under constant speed and constant elasticity assumption in this chapter. It shows that the difficulty of hypersonic flight control is very big.

\section{References}

[1]A. E. Finzi, M. Lavagna, A. Di Gregorio, Atmospheric re-entry trajectory tracking and control for an unmanned space vehicle with a Lyapunov approach [A], In: AIAA Guidance, Navigation, and Control Conference and Exhibit [C], Austin: AIAA, 2003-5441

[2]Vaddi S S, Sengupta P. Controller design for hypersonic vehicles accommodating nonlinear state and control constraints, In: AIAA Guidance, Navigation, and Control Conference Chicago, USA, AIAA 2009-6286: 1-19.

[3]Christopher I. Marrison. synthesis of robust control systems for a hypersonic aircraft decision and contro1. IEEE, 1994。4:3324-3329

[4]Kevin. P. Groves, Andrea. Serranti, Stephen. Yurkovich. Anti-Windup Control for an 
Air-breathing Hypersonic Vehicle Model. AIAA, 2006-6557: 1-14.

[5]Lohsoonthorn P, Jonckheere E and Dalzell S. Eigenstructure vs constrained $\infty \mathrm{H}$ design for hypersonic winged cone [J]. Journal of Guidance, Control, and Dynamics, 2001, 24(4): 648-658.

[6]Heller M, Holzapfel F and Sachs G. Robust lateral control of hypersonic vehicles [A]. In: AIAA Guidance, Navigation, and Control Conference and Exhibit [C]. Denver, CO, AIAA 2000-4248.

[7]Appleby B D and Adams N J. Robust estimator design applied to the lateral dynamics of a hypersonic vehicle [A]. In: AIAA Guidance, Navigation, and Control Conference and Exhibit [C]. AIAA-1991-2689.

[8]D.K.Schmidt. Ingegrated Control of Hypersonic Vehicles-A Necessity Not Just a Possibility. AIAA,1993:539-549.

[9]Chavez F R and Schmidt D K.Flight dynamics and control of elastic hypersonic vehicles-Modeling uncertainties[A].In:AIAA Guidance, Navigation and Control Conference[C]. Washington,DC, AIAA 94-3629

[10]Chavez F R and Schmidt D K.Uncertainy modeling for multivariable-control robustness analysis of elastic high-speed vehicles[J].Journal of Guidance, Control,and Dynamics, 1999,22(1):87-95. 